\section{Compression and deep vein thrombosis}

\section{Hugo Partsch \\ Department of Dermatology and Angiology, Medical University of Vienna, Vienna, Austria}

\section{Introduction}

Compression may have two beneficial effects in deep vein thrombosis (DVT): i) long term use may reduce the incidence and severity of a post-thrombotic syndrome (PTS); ii) in the acute stage it alleviates pain and swelling.

\section{Reduction of post-thrombotic syndrome}

Based on meta-analyses wearing of com pression stockings after DVT for 2 years reduces the incidence of PTS. ${ }^{1,2}$

Also previous American College of Chest Physicians (ACCP) guidelines have recommended compression after DVT for this indication. ${ }^{3}$ This suggestion has recently been changed, mainly due to the outcome of the SOX trial, which was unable to find a reduction of PTS, 2 years after DVT, when compression stockings were used in comparison to placebo stockings. ${ }^{4}$ This publication caused lot of discussions, in which especially the poor compliance wearing the stockings had been criticized. $^{5}$

However, the most recent ACCP guideline states: In patients with acute DVT of the leg, we suggest not using compression stockings routinely to prevent PTS (Grade 2B). Remarks: this recommendation focuses on prevention of the chronic complication of PTS and not on the treatment of symptoms. For patients with acute or chronic symptoms, a trial of graduated compression stockings is often justified. ${ }^{6}$

It may be assumed that future guidelines will advocate a tailored regime suggesting compression for symptomatic patients as soon as data will be available. ${ }^{?}$

\section{Reduction of pain and swelling in the acute stage}

Starting compression in the acute stage of DVT, at the same time as anticoagulation is a real Cinderella indication, which tends to fall into oblivion.

The description that strongly applied inelas- tic Unna boot bandages (zinc paste invented by Unna) lead to an immediate reduction of pain dates back more than 100 years ago. ${ }^{8}$ Also swelling is immediately reduced, so that mobile patients are able to keep on walking. Several famous European phlebologists followed the recommendation of treating DVT by compression in addition to anticoagulation (G. Bauer, Sweden; R. Tournay, France; K. Sigg, Switzerland; F. Haid-Fischer, Germany). However, this regime was based on experience only and not supported by scientific evidence. The introduction of low molecular heparin (LMWH) allowing safe and efficient anticoagulation by subcutaneous injections of fixed doses and avoiding intravenous infusions opened the possibility for home therapy. In our hands patients could stay ambulant without bed-rest due to the pain relieving effect of simultaneously applied good compression, without the risk of major pulmonary embolism.

A randomized controlled three-arms study was started comparing bed-rest and no compression with compression stockings and with inelastic Fischer bandages in patients with proximal DVT. Since most patients did not want to take the risk of bed-rest and refused to sign the consent for the study plan, the study had to be stopped after 53 patients. ${ }^{10}$ All patients were mobile and received therapeutic doses of LMWH and overlapping vitamin $\mathrm{K}$ antagonists. One group received firmly applied Unna-boot bandages (Fischer-bandages) ${ }^{8}$ the second group, thigh-length compression stockings, and the third group had bed-rest and no compression. Both compression groups were encouraged to walk. Study duration was 10 days, starting on the day of admission to the hospital because of verified DVT extending into the thigh $(\mathrm{n}=37)$ or pelvis $(\mathrm{n}=16)$.

Pain was assessed by visual analogue scale and by a modified Lowenberg-test. (Assessing the tolerability of a pressure applied to both calves using a blood pressure cuff the difference between the tolerated pressure on the non-affected limb minus that on the leg with DVT gives a quantifiable parameter for pain). In addition, daily walking distances, quality of life and differences of leg circumferences were measured. Repeated lung scans were performed as a safety feature.

The firmly applied zinc paste bandages were wrapped over by inelastic textile bandages (Rosidal K®; Lohmann \& Rauscher AG, St. Gallen, Switzerland), and adhesive bandages were applied over the knee and thigh up to the groin (Panelast ${ }^{\circledR}$ and Porelast $\AA$; Lohmann \& Rauscher AG). The initial resting pressure on the leg was $>50 \mathrm{mmHg}$. These bandages stayed overnight and were changed every 2-3 days. Thigh length compression stockings (23-31 $\mathrm{mmHg}$ ) (Sigvaris 503; Sigvaris Management AG, Winterthur, Switzerland) were also worn
Correspondence: Hugo Partsch, Department of Dermatology and Angiology, Medical University of Vienna, Baumeistergasse 85, A 1160 Vienna, Austria.

E-mail: Hugo.Partsch@meduniwien.ac.at

This work is licensed under a Creative Commons Attribution 4.0 License (by-nc 4.0)

(C) Copyright H. Partsch, 2016

Licensee PAGEPress, Italy

Veins and Lymphatics 2016; 5:5991

doi:10.4081/vl.2016.5991

during night.

In spite of the low number of recruited patients the outcome was convincing. There were statistically significant superior results for pain, swelling and quality of life favoring the compression groups in comparison to bed rest and no compression. Globally, no significant difference was found between the efficacy of stockings and bandages. Pain reduction was much faster with compression compared to bed-rest. One day after starting with inelastic Fischer-bandages the pain level was in the same range as in the bed rest group after 5 days.

More randomized clinical trials regarding the value of compression are rare. A study, concentrating on the post-thrombotic sequelae, after one year reported a faster reduction of clinical symptoms including pain and swelling if strong bandages were applied for one week in the acute phase of DVT in contrast to no compression. ${ }^{11}$

Recently the authors of the SOX trial have published a sub-analysis of their data concentrating on their findings after one month, concluding that compression stockings do not reduce leg pain in patients with acute proximal DVT. ${ }^{12}$ However, this conclusion is invalid, since treatment with compression started only 2-3 weeks after the onset of DVT, at which time acute symptoms have already disappeared. ${ }^{13}$

In conclusion new data are supporting the experience that good compression applied immediately is able to reduce pain and edema in DVT patients. Future randomized trials should start in the acute phase of compression since we know that the clinical outcome in this acute phase will have a deciding influence on the development of PTS.

\section{References}

1. Musani MH, Matta F, Yaekoub AY, et al. Venous compression for prevention of postthrombotic syndrome: a meta-analysis. Am J Med 2010;123:735-40.

2. Tie HT, Luo MZ, Luo MJ, et al. 
Compression therapy in the prevention of postthrombotic syndrome: a systematic review and meta-analysis. Medicine (Baltimore) 2015;94:e1318.

3. Kearon C, Akl EA, Comerota AJ, et al. Antithrombotic therapy for VTE disease: antithrombotic therapy and prevention of thrombosis, 9th ed: american college of chest physicians evidence-based clinical practice guidelines. Chest 2012;141:e419S-94S.

4. Kahn SR, Shapiro S, Wells PS, et al. SOX trial investigators. Compression stockings to prevent post-thrombotic syndrome: a randomised placebo-controlled trial. Lancet 2014;383:880-8.

5. Labropoulos N, Gasparis AP, Caprini JA, Partsch H. Compression stockings toprevent post-thrombotic syndrome. Lancet
2014;384:129-30.

6. Kearon C, Akl EA, Omelas E, et al. Antithrombotic therapy for VTE disease: CHEST guideline and expert panel report. Chest 2016;149:315-52.

7. Ten Cate-Hoek AJ, Ten Cate H, Tordoir J, et al. Individually tailored duration of elastic compression therapy in relation to incidence of the postthrombotic syndrome. J Vasc Surg 2010;52:132-8.

8. Fischer H. Eine neue Therapie der Phlebitis. Med Klinik 1910;30.

9. Partsch H. Therapy of deep vein thrombosis with low molecular weight heparin, leg compression and immediate ambulation. Vasa 2001;30:195-204.

10. Blättler W, Partsch H. Leg compression and ambulation is better than bed rest for the treatment of acute deep venous thrombo- sis. Int Angiol 2000;22:393-400.

11. Roumen-Klappe EM, den Heijer M, van Rossum J, et al. Multilayer compression bandaging in the acute phase of deep-vein thrombosis has no effect on the development of the post-thrombotic syndrome. J Thromb Thrombolysis 2009;27:400-5.

12. Kahn SR, Shapiro S, Ducruet T, et al. Graduated compression stockings to treat acute leg pain associated with proximal DVT. A randomised controlled trial. Thromb Haemost 2014;112:1137-41.

13. Partsch H. Pain relief by compression in acute DVT. A critique of Kahn et al. Thromb Haemost 2014; 112: 1137-1141. Thromb Haemost 2015;113:9067. 